in the field of biological inquiry both emergent and resultant advance. My plea is for careful analysis.

There are a good many critics who seem not yet to have grasped just where the concept of emergent evolution is applicable. They seek to apply it where I, for one, hold it to be inapplicable. 'They may then ask: What bearing has this concept of emergence on the theory of natural selection? It may savour of extravagance if I express the opinion that on this theory, as such, it has little or no bearing.

To make my meaning clear, I must ask : Are we, under natural selection, dealing with the survival of variants or with the origin and transmission of variations? In the opinion I express I assume that the theory of natural selection as such deals with variants, and that the origin and transmission of variations fall for discussion under a different theory-that of genetics. If this be so, the issue for natural selection is a plain issue. Are some variants weeded out in 'the struggle for existence' or are they not? If some are weeded out, leaving others to survive, I regard such elimination as a resultant effect.

That leaves the origin of variations (or of mutations) to be discussed as a separate issue under genetics. It opens up a wide field of inquiry, including Mendelian research. Here the question does arise : Is this or that variant the outcome of resultant, or emergent, advance; or is it a joint product of both? If both are given in the evidence, the emergent factors should be distinguished.

My plea is: If the concept of emergence be accepted, let us make quite clear just where this concept is applicable. When I express the opinion that it is not applicable to natural selection, as such, it should be obvious that this does not preclude the survival of those variants which have genetic characters that can be shown, under searching analysis, to be emergent in origin. Biological inquiry includes both natural selection and genetics; and genetics discloses, as I think, both emergents and resultants. Is there not pressing need for the exercise of distinguishing analysis?

\title{
Fat-soluble Vitamins.
}

$\mathrm{B}^{\mathrm{A}}$ ARELY two decades have elapsed since the concept of 'vitamins' first began seriously to attract the attention of investigators. Scurvy had been recognised as a clinical entity for a couple of centuries, and the treatment of it, by means of fresh vegetables and fruit juices, was well known. But the idea that disease might be caused by the deficiency of some factor in the diet was, for many, too novel to be accepted without question, and much work was necessary before the reality of the accessory food factors or vitamins was generally admitted. Recognised at first solely by the effects produced on experimental animals when absent from their carefully purified diets, it was not long before chemical investigations began to define their properties, from which tentative conclusions as to their chemical nature might be drawn. With the discovery that ultra-violet light could cure rickets, and was also capable of making a diet, previously inactive, protective against this disease, a new key was provided for the unlocking of the door which led to the chemical constitution of the anti-rachitic vitamin, or vitamin $\mathrm{D}$, as it is also called. At this stage the work came into contact with other investigations on a group of compounds of widespread distribution in Nature, but of almost unknown biological significance, the sterols. At the present time it is certain that vitamin $\mathrm{D}$, if not actually a member of this group, is closely related to one, and it is extremely probable that vitamin A, or the fat-soluble growth-promoting vitamin, is also of a similar nature.

Following the discovery that exposure to ultraviolet light could render a deficient diet antirachitic, it was soon found that the unsaponifiable fraction of the fat of the diet was responsible for this effect. O. Rosenheim and T. A. Webster, working at the National Institute for Medical Research, and Steenbock and Hess and their collaborators in America, then discovered independently that 'chemically pure' cholesterol was rendered anti-rachitic by this exposure. Further work by these and other investigators has now sufficed to determine more definitely the properties and nature of the compound which undergoes this change, although the actual nature of the change itself is undetermined.

Rosenheim and Webster (Biochem. Jour., 1926, vol. 20 , p. 537 ; Lancet, 1927, vol. 1, p. 306) were unable to convert more than 0.1 per cent. of cholesterol into vitamin $\mathrm{D}$ under the influence of ultra-violet light. They also showed that the presence of the unsaturated linkage and of the secondary alcohol group of the sterol was essential for the reaction to take place, and that the vitamin was not precipitable by digitonin. The fact that only a minute amount of the cholesterol could be 'activated' raised a doubt as to whether this substance was the true precursor of vitamin $\mathrm{D}$, and the doubt became a certainty when it was found that cholesterol purified by way of the dibromide could not be activated and, moreover, no longer possessed the characteristic absorption spectrum in the ultra-violet region. These experiments proved that the vitamin precursor is not cholesterol itself, but some substance which is closely associated with it when obtained from all natural sources.

Further work showed that the precursor was easily oxidised and could also be precipitated by digitonin, unlike the vitamin obtained from it. Attempts to separate it from cholesterol by making use of the latter property, or by fractional crystal. lisation (Heilbron, Kamm, and Morton, Jour. Soc. Chem. Ind., 1926, vol. 45 , p. 932) or by fractional distillation in a high vacuum (Windaus), resulted in a considerable concentration of the precursor, but it was not obtained in a pure state.

No. 3004, VoL. 119] 
The authors therefore selected another sterol, ergosterol, which they had previously shown could be rendered anti-rachitic by ultra-violet light, for further examination, since it possesses some of the properties of the vitamin precursor: thus, it cannot be recovered unchanged from its bromide (Windaus), it is extremely sensitive to light and oxidation, forms an insoluble digitonide, and possesses three unsaturated linkages. It was found that this sterol exhibited a very pronounced absorption in the ultra-violet region of the spectrum, which disappeared on irradiation; at the same time the product lost the property of being precipitated by digitonin. Experiments on rats suggested that the limit of anti-rachitic activity will be in the region of a daily dose of $\frac{1}{1000} \mathrm{mgm}$. or less.

It is therefore probable that the vitamin precursor is really ergosterol: similar or identical sterols have been found widely distributed throughout the lower plants. Thence they must find their way into animals, thus enabling the latter to develop their own anti-rachitic vitamin on exposure to light. The anti-rachitic power developed by cholesterol on irradiation, then, is due to contamination of this compound with ergosterol ; from the intensity of the ultra-violet absorption spectrum it appears that this contamination occurs to the extent of about 0.05 per cent. : this assumption also explains the impossibility of making anti-rachitic more than a small amount of the "cholesterol."

Less is known at the moment about the nature of the fat-soluble vitamin A. Like vitamin D, which has only recently been definitely differentiated from it, it occurs in the unsaponifiable fraction of the fats and oils (notably codliver oil) in which it is present. Following the work of Drummond, Takahashi, and other investigators, certain of its properties have become established (see Nature, 1926, vol. 117, p. 522, and J. C. Drummond, H. J. Channon, and K. H. Coward, Biochem. Jour., 1925, vol. 119, p. 1047). Thus the vitamin A present in the cholesterol-free oil obtained from the unsaponifiable matter of codliver oil can be distilled at low pressure at a temperature of about $180^{\circ}-220^{\circ} \mathrm{C}$. : only small amounts are obtained, the greater part of the purified oil consisting of unsaturated alcohols together with a certain amount of squalene. Even the product obtained in this manner is impure, so that Drummond was unable to agree with Takahashi with reference to the claim advanced by the latter that he had isolated the vitamin in a pure state. Drummond noticed that the growthpromoting activity was retained after destruction of the hydroxyl group of the alcohols present, but was always destroyed by exposure to reagents which affected the unsaturated linkages. More recently Rosenheim (Med. Res. Council Rep., 1925-6, p. 30) has found that partial oxidation of cholesterol produces a substance which gives the colour reaction characteristic of vitamin A (and a few other substances). It is therefore possible that vitamin A may also be found to be a derivative of one of the sterols.
The story of the work on the fat-soluble vitamins $A$ and $D$ shows how two entirely different lines of research may suddenly converge, and how a purely academic investigation may suddenly assume a definite practical importance. Without the knowledge obtained from the work of previous investigators on the sterols, the biological importance of which was not at the time obvious, it is possible that the isolation of these two vitamins would have taken much longer than it now seems probable will be the case.

The practical application of these researches in the prevention and cure of rickets is obvious : but this is not a very common disease, so that it is worth inquiring if deficiency of these vitamins may play a part in other diseases or states of ill-health. Drawing an analogy from the symptoms shown by rats suffering from deficiency of vitamin $A$, it is probable that this vitamin plays a part in maintaining the various mucous membranes of the body in a healthy state, quite apart from any effect it has on proper growth : in fact, deficiency of this vitamin has led to the appearance of xerophthalmia in human beings just as in experimental animals.

At the same time, increasing light is being thrown on the relationship between vitamin $D$ and the formation and decay of the teeth, a subject of very great importance. Mrs. Mellanby first demonstrated this relationship in the case of animals, but the application to human beings was not immediately obvious, since dental decay occurs frequently in apparently perfectly formed teeth. Mrs. Mellanby has, however, found (Med. Res. Council Rep., 1925-6, pp. 18 and 74) that teeth, normal to naked-eye examination, may show defects of structure of both the enamel and dentine when examined microscopically. Decay was almost general in these teeth, whilst only one-quarter of the well-formed teeth were affected.

With C. I. Pattison, Mrs. Mellanby has investigated the extension of caries in children on different diets, and has found that increasing the vitamin $D$ and decreasing the oatmeal has reduced the extension, when compared with less satisfactorily constructed diets.

The fact that apparently well-nourished people give signs, in the structure of their teeth, of a specific vitamin deficiency in their diet, is of great interest. It suggests that the supply of vitamin has been inadequate, and that in the competition for the amount provided in the diet, the teeth fail to obtain their due share. This work brings the problem of dental decay within the sphere of nutrition, and points the way to effective prevention: either the diet must be improved by the increased use of natural foodstuffs containing the vitamin, or a palatable and cheap source of the vitamin must be available. The work on the irradiation of ergosterol gives grounds for suggesting that it may be possible to provide, in the near future, vitamin $D$ in a highly concen. trated form, to be used either by itself as a medicament or for the enrichment of suitable articles of diet.

No. 3004, VoL. 119] 